\title{
Global Stability of an Epidemic Model of Computer Virus
}

\author{
Xiaofan Yang, ${ }^{1,2}$ Bei Liu, ${ }^{1}$ and Chenquan Gan ${ }^{1,2}$ \\ ${ }^{1}$ School of Electronic and Information Engineering, Southwest University, Chongqing 400715, China \\ ${ }^{2}$ College of Computer Science, Chongqing University, Chongqing 400044, China
}

Correspondence should be addressed to Chenquan Gan; gcq2010cqu@163.com

Received 5 July 2014; Accepted 27 August 2014; Published 15 October 2014

Academic Editor: Claudio Cuevas

Copyright (C) 2014 Xiaofan Yang et al. This is an open access article distributed under the Creative Commons Attribution License, which permits unrestricted use, distribution, and reproduction in any medium, provided the original work is properly cited.

\begin{abstract}
With the rapid popularization of the Internet, computers can enter or leave the Internet increasingly frequently. In fact, no antivirus software can detect and remove all sorts of computer viruses. This implies that viruses would persist on the Internet. To better understand the spread of computer viruses in these situations, a new propagation model is established and analyzed. The unique equilibrium of the model is globally asymptotically stable, in accordance with the reality. A parameter analysis of the equilibrium is also conducted.
\end{abstract}

\section{Introduction}

The past few decades have witnessed the overflow of computer viruses. What is more serious is that enormous financial losses and social panic have also been caused by them [1]. Consequently, it is necessary to study the propagation behavior of computer viruses and to explore some effective defense measures. Undoubtedly, dynamical modeling is one of the most effective approaches.

To portray the important feature of computer viruses that, in general, an infected computer has infectivity during its latent period, Yang et al. [2] proposed an SLBS (susceptible-latent-breaking-susceptible) model, in which infected computers are divided into two groups: $L$ computers and $B$ computers, and every $L$ computer possesses infectivity.

Based on this model and the fact that, in general, an infected computer would possess temporary immunity when it is cured by installing the latest version of antivirus software, an SLBRS (susceptible-latent-breaking-recoveredsusceptible) model (see Figure 1) is studied in [3].

Unfortunately, there are three main flaws in this model. On one hand, this model ignores the effect of infected computers connected to the Internet. Due to the fact that no antivirus software can detect and clear all sorts of computer viruses [4] and computers can enter or leave the Internet easily, the entering computers should not be regarded as susceptible completely. On the other hand, this model neglects the impact of antivirus software. With the rapid advance of computer and communication technologies, it has never been easier to obtain the latest version of antivirus software. This implies that every susceptible or latent computer can be recovered directly. Finally, this model overlooks the influence of reinstalling the operating system. In reality, operating systems often need to be reinstalled, under which the latent or breaking computer would become susceptible.

To overcome the above mentioned defects, a new reasonable model (see Figure 2) is investigated in this paper. The existence and global stability of the equilibrium are not only proved by mathematical analysis but also displayed by numerical simulation. A parameter analysis of the equilibrium is also included.

The rest of this paper is structured as follows. Section 2 formulates the new model. Section 3 mainly considers the global stability of the equilibrium. Finally, Section 4 summarizes this work. 


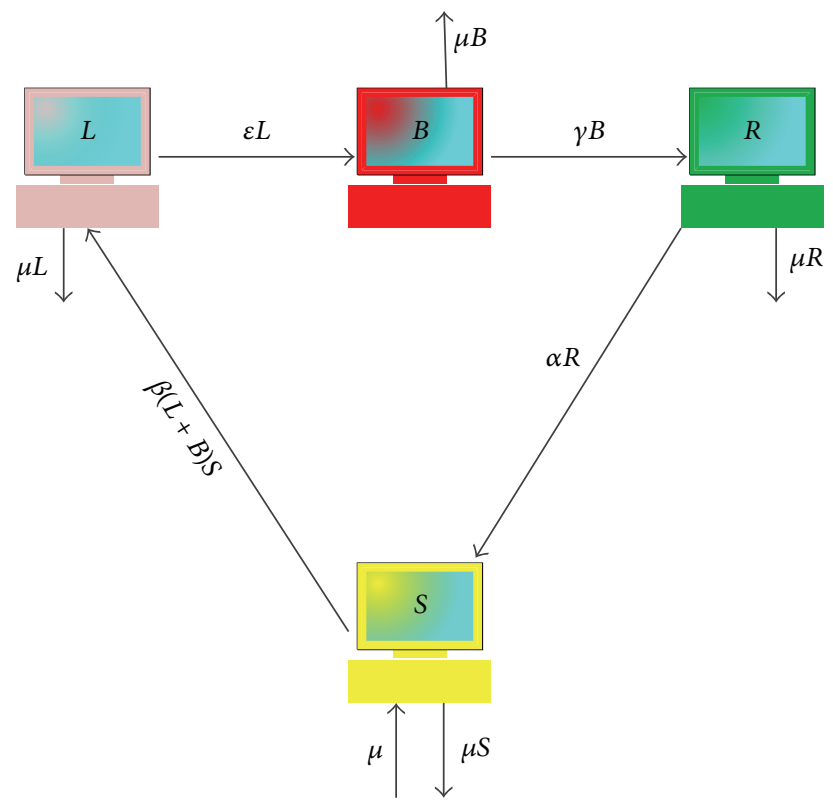

FIGURE 1: The transfer diagram of the SLBRS model.

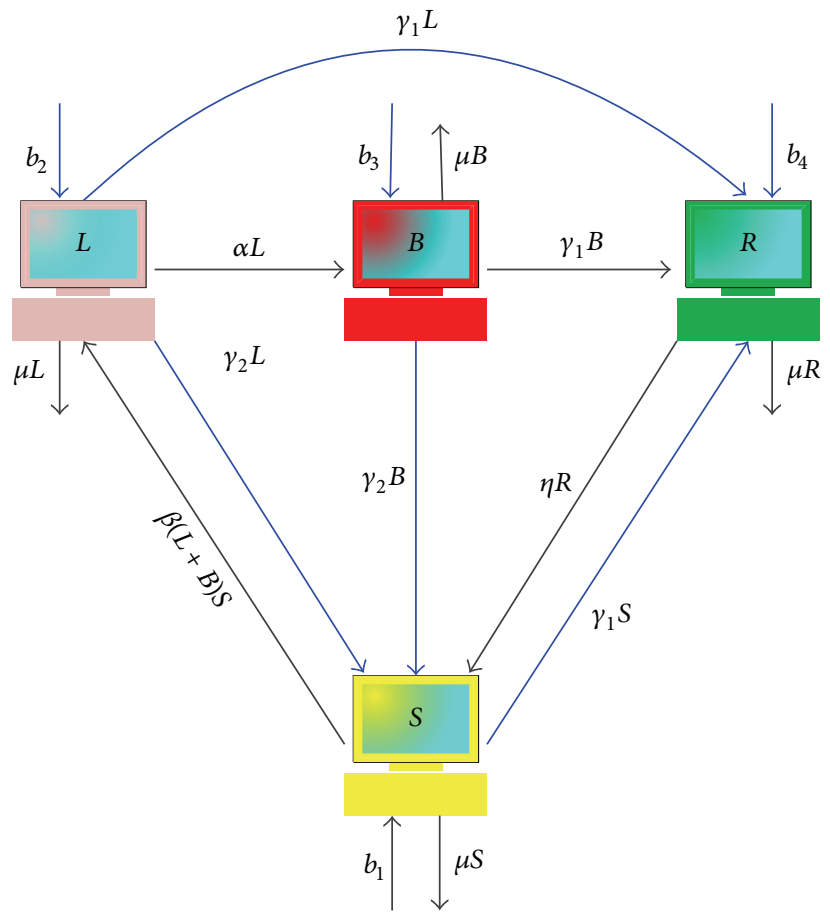

FIGURE 2: The transfer diagram of the new SLBRS model.

\section{Model Formulation}

Applying the same model assumptions in [3] to the new $S L B R S$ model, it follows from Figure 2 that the corresponding differential system is

$$
\begin{gathered}
\dot{S}=b_{1}+\gamma_{2} L+\gamma_{2} B+\eta R-\gamma_{1} S-\mu S-\beta(L+B) S, \\
\dot{L}=b_{2}+\beta(L+B) S-\gamma_{1} L-\gamma_{2} L-\mu L-\alpha L,
\end{gathered}
$$

$$
\begin{gathered}
\dot{B}=b_{3}+\alpha L-\gamma_{1} B-\gamma_{2} B-\mu B, \\
\dot{R}=b_{4}+\gamma_{1} L+\gamma_{1} B+\gamma_{1} S-\mu R-\eta R,
\end{gathered}
$$

where $S, L, B$, and $R$ represent, at time $t$, the average numbers of susceptible, latent, breaking, and recovered computers, 
respectively. Their entering rates are $b_{1}, b_{2}, b_{3}$, and $b_{4}$, respectively. The explanations of the rest of parameters are as follows.

(i) $\alpha$ : the breaking rate.

(ii) $\beta$ : the incidence rate.

(iii) $\gamma_{1}$ : the recovered rate.

(iv) $\mu$ : the rate of a computer leaving the Internet.

(v) $\eta$ : the rate of a recovered computer losing immunity.

(vi) $\gamma_{2}$ : the rate of an infected computer reinstalling the operating system.

Let $N=S+L+B+R, b=b_{1}+b_{2}+b_{3}+b_{4}, N^{*}=$ $b / \mu$, and $R^{*}=\left(\gamma_{1} N^{*}+b_{4}\right) /\left(\gamma_{1}+\mu+\eta\right)$. Then, adding up the four equations of system (1) and simplifying, one can get $d N(t) / d t=b-\mu N(t)$, implying $\lim _{t \rightarrow+\infty} N(t)=N^{*}$. Similarly, $\lim _{t \rightarrow+\infty} R(t)=R^{*}$. Thus, system (1) can be written as the limiting system [5]:

$$
\begin{aligned}
\dot{L}= & b_{2}-\left(\gamma_{1}+\gamma_{2}+\alpha+\mu\right) L \\
& +\beta(L+B)\left[\left(N^{*}-R^{*}\right)-(L+B)\right], \\
& \quad \beta=b_{3}+\alpha L-\left(\gamma_{1}+\gamma_{2}+\mu\right) B .
\end{aligned}
$$

In what follows, we will consider the global behavior of system (2) with respect to the positively invariant region: $\Omega=$ $\left\{(L, B) \in R_{+}^{2}: L+B \leq N^{*}\right\}$.

\section{Theoretical Analysis}

\subsection{Equilibrium}

Theorem 1. System (2) has a unique equilibrium $E^{*}\left(L^{*}, B^{*}\right)$, where

$$
\begin{gathered}
w_{0}=\beta\left(N^{*}-R^{*}\right)-\left(\gamma_{1}+\gamma_{2}+\mu\right), \\
B^{*}=\frac{2 \beta b_{3}+\alpha\left[w_{0}+\sqrt{w_{0}^{2}+4 \beta\left(b_{2}+b_{3}\right)}\right]}{2 \beta\left(\gamma_{1}+\gamma_{2}+\mu+\alpha\right)}, \\
L^{*}=\frac{\left(\gamma_{1}+\gamma_{2}+\mu\right) B^{*}-b_{3}}{\alpha} .
\end{gathered}
$$

Proof. Assume that $(\widetilde{L}, \widetilde{B})$ is an equilibrium of system (2). Then,

$$
\begin{gathered}
b_{2}-\left(\gamma_{1}+\gamma_{2}+\alpha+\mu\right) \widetilde{L}+\beta(\widetilde{L}+\widetilde{B}) \\
\times\left[\left(N^{*}-R^{*}\right)-(\widetilde{L}+\widetilde{B})\right]=0, \\
b_{3}+\alpha \widetilde{L}-\left(\gamma_{1}+\gamma_{2}+\mu\right) \widetilde{B}=0 .
\end{gathered}
$$

Adding up the two equations of system (4), one can obtain

$$
\beta(\widetilde{L}+\widetilde{B})^{2}-w_{0}(\widetilde{L}+\widetilde{B})-\left(b_{2}+b_{3}\right)=0,
$$

implying that

$$
\widetilde{L}+\widetilde{B}=\frac{w_{0}+\sqrt{w_{0}^{2}+4 \beta\left(b_{2}+b_{3}\right)}}{2 \beta} .
$$

From (6) and the second equation of system (4), one can get $\widetilde{B}=B^{*}, \widetilde{L}=L^{*}$. Thus, the proof is complete.

Corollary 2. Let $I^{*}=L^{*}+B^{*}$. Then, $\partial I^{*} / \partial \beta>0, \partial I^{*} / \partial \eta>0$, $\partial I^{*} / \partial b_{1}>0, \partial I^{*} / \partial b_{2}>0, \partial I^{*} / \partial b_{3}>0, \partial I^{*} / \partial b_{4}>0$, $\partial I^{*} / \partial \gamma_{1}<0, \partial I^{*} / \partial \gamma_{2}<0$, and $\partial I^{*} / \partial \mu<0$.

Proof. Let $w=\left(w_{0}+\sqrt{w_{0}^{2}+4 \beta\left(b_{2}+b_{3}\right)}\right) / \sqrt{w_{0}^{2}+4 \beta\left(b_{2}+b_{3}\right)}$. As $L^{*}+B^{*}=\left(w_{0}+\sqrt{w_{0}^{2}+4 \beta\left(b_{2}+b_{3}\right)}\right) / 2 \beta$, then

$$
\begin{gathered}
\frac{\partial I^{*}}{\partial \beta}=\left(N^{*}-R^{*}\right) w+\frac{2\left(b_{2}+b_{3}\right)}{\sqrt{w_{0}^{2}+4 \beta\left(b_{2}+b_{3}\right)}}>0, \\
\frac{\partial I^{*}}{\partial \eta}=\frac{w\left(\gamma_{1} N^{*}+b_{4}\right)}{2\left(\gamma_{1}+\mu+\eta\right)^{2}}>0, \\
\frac{\partial I^{*}}{\partial b_{1}}=\frac{w(\mu+\eta)}{2 \mu\left(\gamma_{1}+\mu+\eta\right)}>0, \\
\frac{\partial I^{*}}{\partial b_{3}}=\frac{w(\mu+\eta)}{2 \mu\left(\gamma_{1}+\mu+\eta\right)}+\frac{1}{\sqrt{w_{0}^{2}+4 \beta\left(b_{2}+b_{3}\right)}}>0, \\
\left.\frac{\partial I^{*}}{\partial \mu}=-w \frac{1}{2 \mu}+\mu+\eta\right) \\
\frac{\mu_{1}^{2}\left(b_{1}+b_{2}+b_{3}\right)+b \eta\left(\gamma_{1}+2 \mu+\eta\right)}{2\left(\gamma_{1}+\mu+\eta\right)^{2} \mu^{2}}-\frac{w}{2 \beta}<0 . \\
\frac{\partial I^{*}}{\partial b_{4}}=\frac{w\left(N^{*}+4 \beta\left(b_{2}+b_{3}\right)\right.}{2 \mu\left(\gamma_{1}+\mu+\eta\right)}>0, \\
2\left(\gamma_{1}+\mu+\eta\right) \\
\frac{\partial I^{*}}{\partial \gamma_{2}}=-\frac{w}{2 \beta}<0,
\end{gathered}
$$

The proof is complete.

Remark 3. Corollary 2 shows the effects of system parameters on the number of infected computers.

\subsection{The Local Stability of the Equilibrium}

Theorem 4. $E^{*}$ is locally asymptotically stable with respect to $\Omega$.

Proof. The Jacobian matrix of system (2) evaluated at $E^{*}$ is

$$
J_{E^{*}}=\left(\begin{array}{cc}
w_{1}-\left(\gamma_{1}+\gamma_{2}+\alpha+\mu\right) & w_{1} \\
\alpha & -\left(\gamma_{1}+\gamma_{2}+\mu\right)
\end{array}\right),
$$


where $w_{1}=\gamma_{1}+\gamma_{2}+\mu-\sqrt{w_{0}^{2}+4 \beta\left(b_{2}+b_{3}\right)}$. The corresponding characteristic equation is

$$
\lambda^{2}+w_{2} \lambda+w_{3}=0
$$

where

$$
\begin{aligned}
& w_{2}=\gamma_{1}+\gamma_{2}+\mu+\alpha+\sqrt{w_{0}^{2}+4 \beta\left(b_{2}+b_{3}\right)}>0, \\
& w_{3}=\left(\gamma_{1}+\gamma_{2}+\mu+\alpha\right) \sqrt{w_{0}^{2}+4 \beta\left(b_{2}+b_{3}\right)}>0 .
\end{aligned}
$$

Hence, it follows from the Hurwitz criterion that the two roots of (9) have negative real parts. The claimed result follows.

3.3. The Global Stability of the Equilibrium. First of all, let us consider the following two lemmas, which are useful in the sequel.

Lemma 5. System (2) has no periodic orbit in the interior of $\Omega$.

Proof. Let

$$
\begin{aligned}
& f_{1}(L, B)= b_{2}-\left(\gamma_{1}+\gamma_{2}+\alpha+\mu\right) L \\
&+\beta(L+B)\left(N^{*}-R^{*}-L-B\right), \\
& f_{2}(L, B)= b_{3}+\alpha L-\left(\gamma_{1}+\gamma_{2}+\mu\right) B, \\
& D(L, B)=\frac{1}{L B} .
\end{aligned}
$$

Then,

$$
\begin{aligned}
& \frac{\partial\left(D f_{1}\right)}{\partial L}+\frac{\partial\left(D f_{2}\right)}{\partial B} \\
& \quad=-\frac{b_{2}}{B L^{2}}-\frac{b_{3}}{L B^{2}}-\frac{\alpha}{B^{2}}-\frac{\beta}{B}-\frac{\beta}{L^{2}}\left(N^{*}-R^{*}-B\right)<0 .
\end{aligned}
$$

Thus, it follows from the Bendixson-Dulac criterion [6] that system (2) admits no periodic orbit in the interior of $\Omega$.

Lemma 6. System (2) has no periodic orbit that passes through a point on $\partial \Omega$, the boundary of $\Omega$.

Proof. Suppose that $(\bar{L}, \bar{B})$ is an arbitrary point on $\partial \Omega$. Then, let us consider the following three possible cases.

Case 1. $0 \leq \bar{L} \leq N^{*}, \bar{B}=0$. Then $\left.\dot{B}\right|_{(\bar{L}, \bar{B})}=b_{3}+\alpha \bar{L}>0$.

Case 2. $0<\bar{B}<N^{*}, \bar{L}=0$. Then $\left.\dot{L}\right|_{(\bar{L}, \bar{B})}=b_{2}+\beta \bar{B}\left(N^{*}-R^{*}-\right.$ $\bar{B})>0$.

Case 3. $\bar{L}+\bar{B}=N^{*}$. Then $\left.(\dot{L}+\dot{B})\right|_{(\bar{L}, \bar{B})}=-b_{1}-b_{4}-\left(\gamma_{1}+\right.$ $\left.\gamma_{2}\right) N^{*}-\beta N^{*} R^{*}<0$

Hence, the claimed result follows from the above discussions.

Now, it is the turn to examine the main result of this paper.

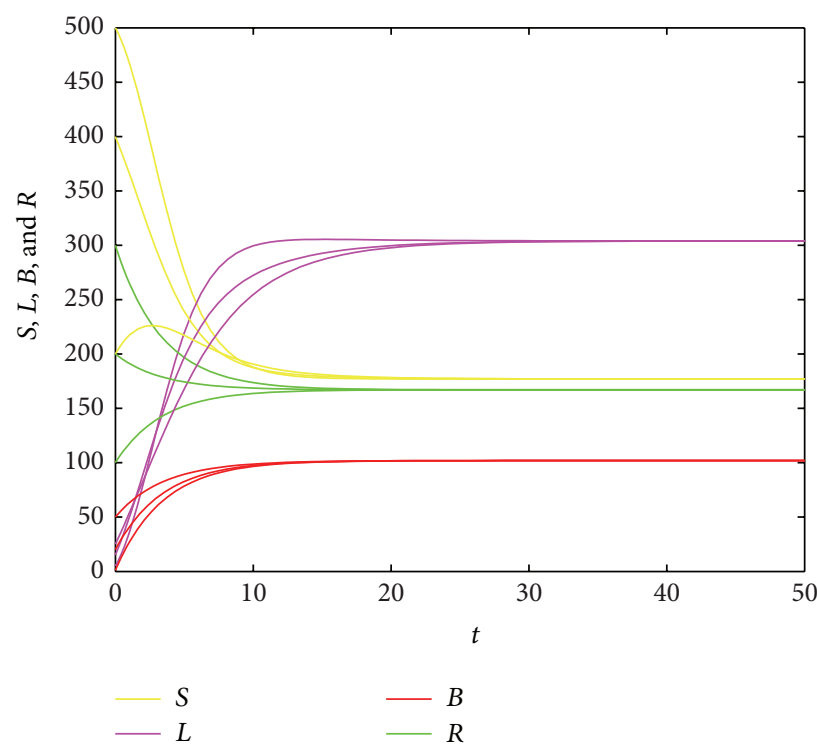

Figure 3: Time plots of $S, L, B$, and $R$ for a common system with three different initial conditions.

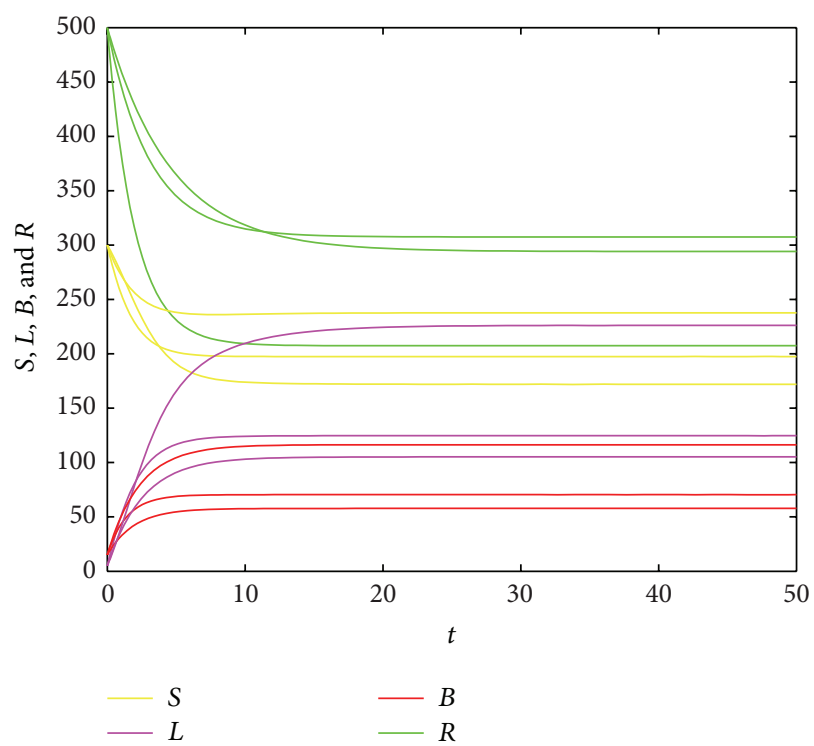

Figure 4: Time plots of $S, L, B$, and $R$ for three different systems with a common initial condition.

Theorem 7. $E^{*}$ is globally asymptotically stable with respect to $\Omega$.

Proof. The claimed result follows by combining Lemmas 5-6 and the generalized Poincare-Bendixson theorem [6].

Remark 8. Theorem 7 implies that computer viruses would persist on the Internet, in agreement with the fact. This result is also displayed by Figures 3 and 4 . 


\section{Summary}

This paper has investigated a new SLBRS model. The global stability of the unique equilibrium of this model has been proved, which is consistent with the fact that computer viruses on the Internet cannot be eradicated. The main result has also been illustrated.

\section{Conflict of Interests}

The authors declare that there is no conflict of interests regarding the publication of this paper.

\section{Acknowledgments}

The authors wish to express sincere gratitude to the anonymous reviewers for careful reading and valuable suggestions. This work is supported by the Natural Science Foundation of China (Grant nos. 11347150 and 10771227) and the Doctorate Foundation of Educational Ministry of China (Grant no. 20110191110022).

\section{References}

[1] P. Szor, The Art of Computer Virus Research and Defense, Addison-Wesley Education Publishers, New York, NY, USA, 2005.

[2] L.-X. Yang, X. Yang, L. Wen, and J. Liu, "A novel computer virus propagation model and its dynamics," International Journal of Computer Mathematics, vol. 89, no. 17, pp. 2307-2314, 2012.

[3] M. Yang, Z. Zhang, Q. Li, and G. Zhang, "An SLBRS model with vertical transmission of computer virus over the Internet," Discrete Dynamics in Nature and Society, vol. 2012, Article ID 925648, 17 pages, 2012.

[4] Z. Zuo, Q. Zhu, and M. Zhou, "On the time complexity of computer viruses," IEEE Transactions on Information Theory, vol. 51, no. 8, pp. 2962-2966, 2005.

[5] H. R. Thieme, "Asymptotically autonomous differential equations in the plane," Rocky Mountain Journal of Mathematics, vol. 24, no. 1, pp. 351-380, 1994.

[6] R. C. Robinson, An Introduction to Dynamical System: Continuous and Discrete, Prentice Hall, New York, NY, USA, 2004. 


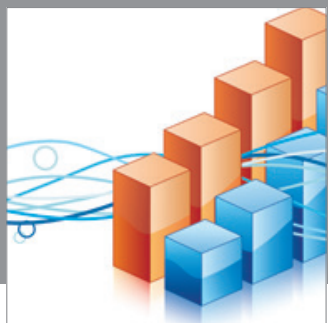

Advances in

Operations Research

mansans

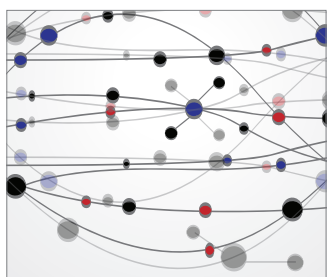

The Scientific World Journal
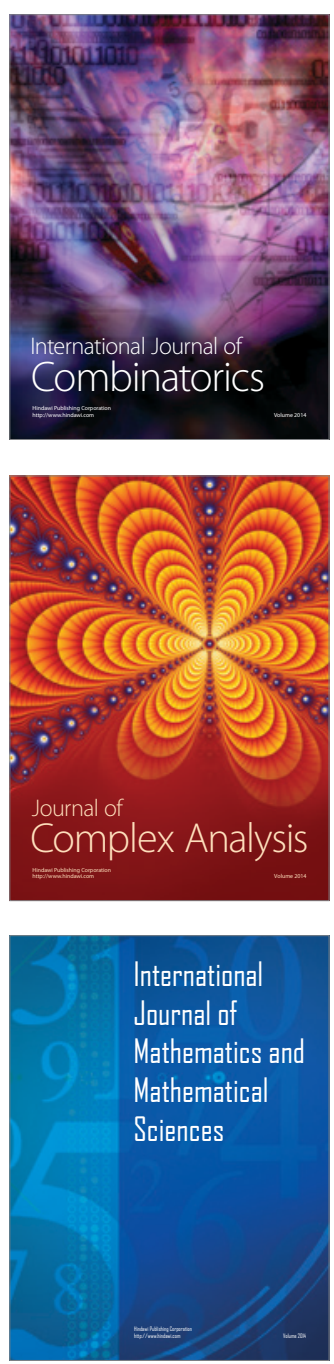
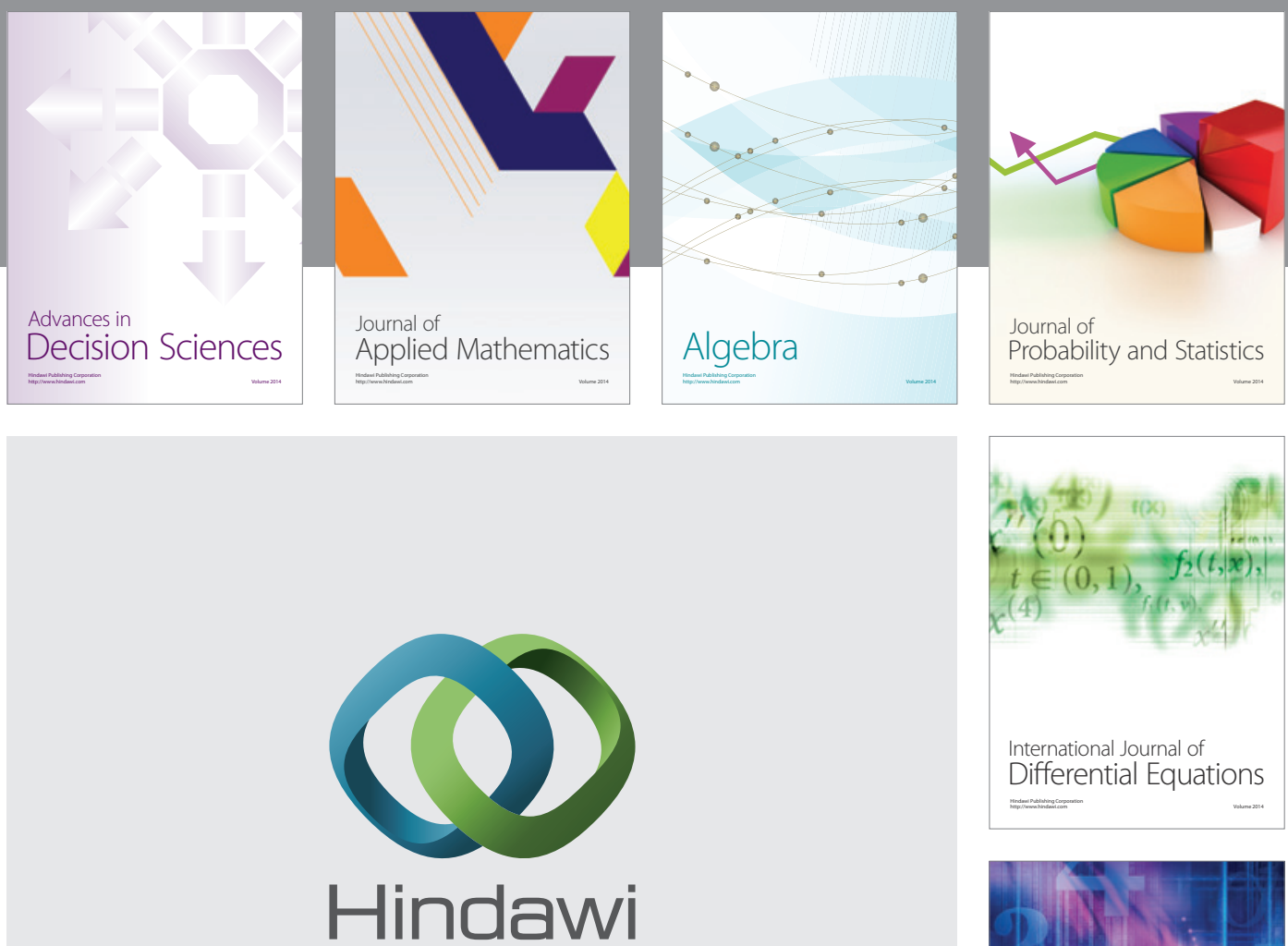

Submit your manuscripts at http://www.hindawi.com
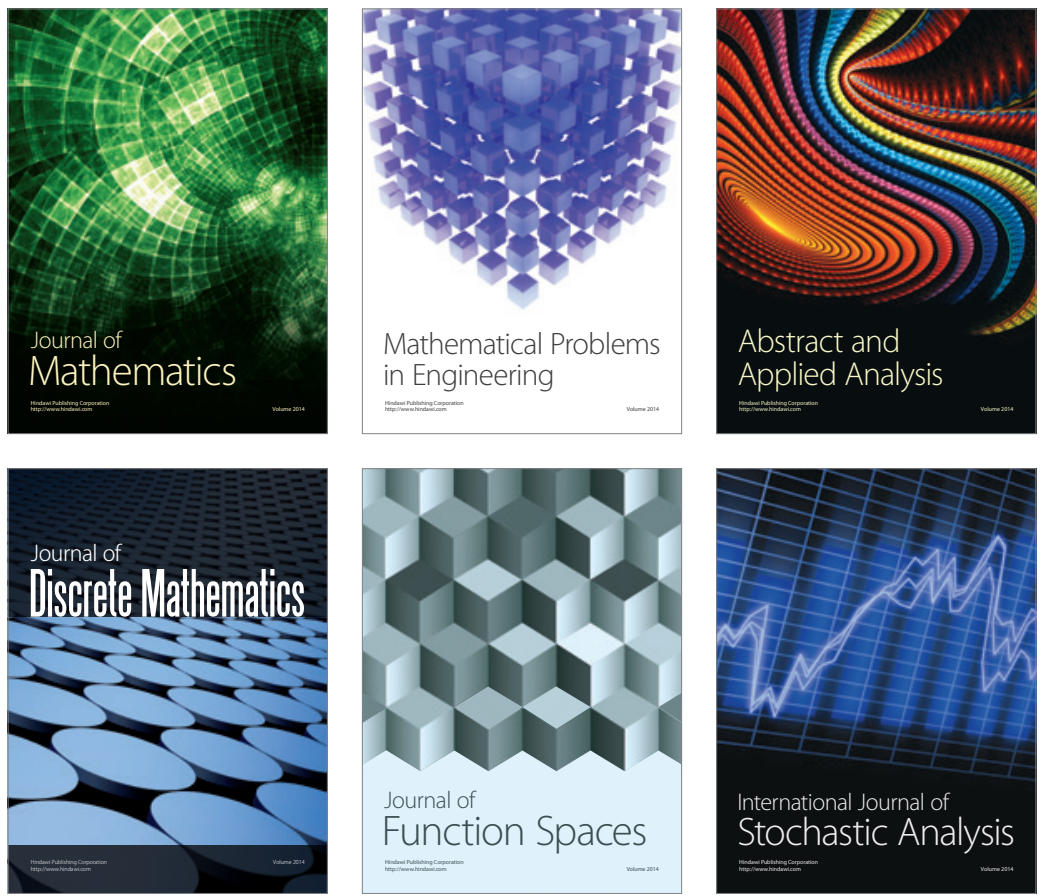

Journal of

Function Spaces

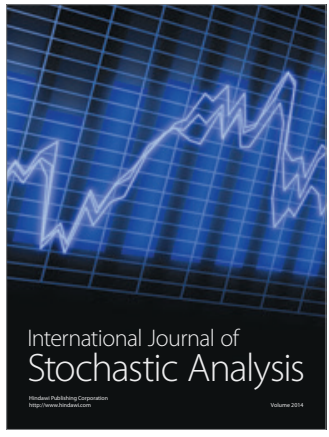

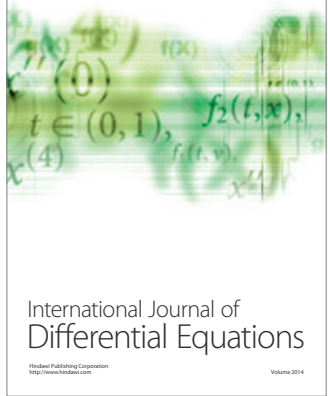
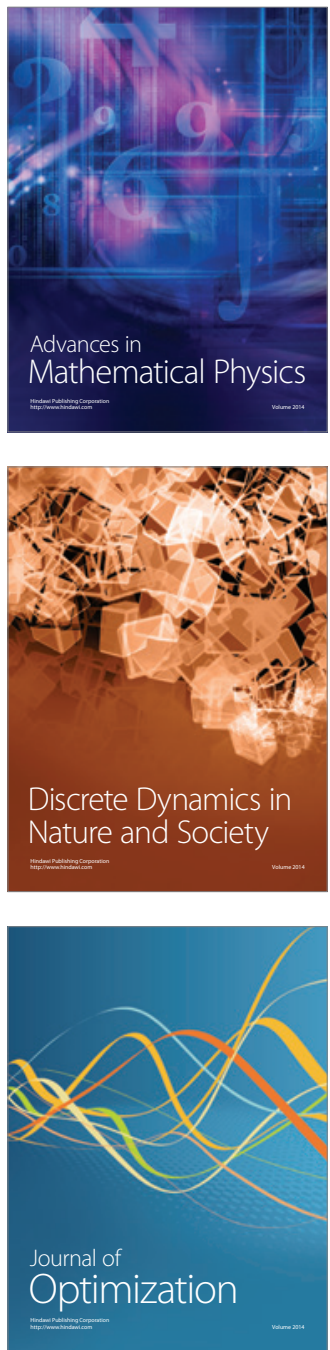